\title{
Análisis de competitividad y movilidad en destinos turísticos del Estado de Guanajuato a través de un modelo de regresión logística
}

Análise da competitividade e da mobilidade em destinos turísticos no estado mexicano de Guanajuato, por meio de um modelo de regressão logística

Analysis of competitiveness and mobility within tourist destinations of Guanajuato through the application of a logistic regression model http://dx.doi.org/10.18472/cvt.17n1.2017.1097

Erick Treviño-Aguilar < erick.trevino@ugto.org > Universidad de Guanajuato (UGTO), Guanajuato, México.

Jeremy Heald <healdj59@gmail.com > Universidad de Guanajuato (UGTO), Guanajuato, México.

Rafael Guerrero-Rodriguez < guerrerorafael1@gmail.com > Universidad de Guanajuato (UGTO), Guanajuato, México.

CRONOLOGIA DO PROCESSO EDITORIAL

Recebimento do artigo: 25-set-2015

Aceite: $27-$ dez-2016

FORMATO PARA CITAÇÃO DESTE ARTIGO

TREVIÑO-AGUILAR, E.; HEALD, J.; GUERRERO-RODRIGUEZ, R. Análisis de competitividad y movilidad en destinos turísticos del Estado de Guanajuato a través de un modelo de regresión logística. Caderno Virtual de Turismo. Rio de Janeiro, v. 17, n. 1, p. 105-122, abr. 2017.

REALIZAÇÃO

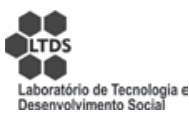

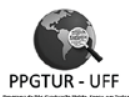

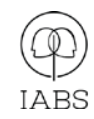

APOIO INSTITUCIONAL

EDIÇÃO

PATROCÍNIO

COPPE

UFR]

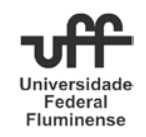

IPBS 


\section{RESUMEN}

Este trabajo presenta una discusión sobre la competitividad y movilidad turística de destinos turísticos en el Estado de Guanajuato, México, en el contexto del turismo cultural. Lo anterior se realiza a través de la aplicación de un modelo de regresión logística para caracterizar la probabilidad de movilidad entre destinos turísticos tradicionales de la entidad y cinco Pueblos Mágicos, previa selección de un conjunto de factores explicativos utilizando datos reales del 2013. A partir de estos datos y la aplicación del modelo, se discuten la teoría y práctica de la competitividad así como la movilidad turística a fin de sugerir algunas recomendaciones respecto del posible desarrollo de alianzas de cooperación entre distintos destinos turísticos del Estado de Guanajuato. La experiencia enseña que el diseño y aplicación del programa es retador y puede proveer aprendizajes para programas similares implementados en diversos países de América Latina.

Palabras clave: Competitividad. Movilidad turística. Regresión logística. Guanajuato, México.

\section{RESUMO}

Este artigo apresenta uma discussão sobre a competitividade e a mobilidade turística dos destinos turísticos no estado de Guanajuato, México, no contexto do turismo cultural. Isso é feito por meio da aplicação de um modelo de regressão logística para caracterizar a probabilidade de mobilidade entre destinos turísticos do estado (os destinos tradicionais e cinco vilas mágicas) mediante a seleção de um conjunto de fatores explicativos usando dados reais do ano de 2013. Baseando-se nesses dados e a aplicação do modelo, são questionadas a teoria e a prática da competitividade, bem como o transporte a fim de sugerir algumas recomendações no que concerne ao eventual desenvolvimento de parcerias e coalizões entre diferentes destinos no estado mexicano de Guanajuato. A experiência ensina que a concepção e implementação do programa é um desafio e a experiência pode fornecer aprendizagems para programas similares implementados em diferentes países da América Latina.

Palavras-chave: Competitividade. Mobilidade turística. Regressão logística. Guanajuato, México.

\section{ABSTRACT}

The article presents a discussion of tourism competitiveness and mobility in tourist destinations in Guanajuato, Mexico in a cultural tourism context. A logistic regression model is employed to characterize the probability of movements between the State of Guanajuato's traditional tourist destinations and five "Pueblos Magicos", selecting a series of explicatory impact factors from 2013 real data. The theory and practice of tourism competitiveness and tourist mobility is discussed with reference to the model in order to make recommendations concerning the possible development of cooperative networks between different tourism destinations in the State of Guanajuato. The experience teaches us that the design and implementation of the program is challenging and can provide wider learning for similar programs in different parts of Latin America.

Palavras clave: Competitivity. Tourism mobility. Logistic regression. Guanajuato, Mexico. 


\section{Introducción}

Las bases de la experiencia de viaje se encuentran principalmente en la idea de movilizar personas de un territorio a otro con un número determinado de motivaciones y consideraciones temporales. En ese sentido, si la experiencia turística depende en gran medida de la movilidad, entonces ésta se convierte en un elemento central en la construcción de la atractividad y la competitividad de los destinos (OLIVERI et al., 2012). Las primeras investigaciones acerca de la movilidad y comportamiento espacial de los turistas se remontan a los años 1970s. La mayoría de estos estudios estaban basados principalmente en análisis de patrones geo-espaciales con una limitada discusión explicativa (SHOVAL; ISSACSON, 2010, p. 11). A pesar de ello, en los últimos años han surgido diferentes enfoques que proponen analizar la dinámica social del territorio derivada del desarrollo de la actividad turística de una manera más integral.

No hay duda que la movilidad siempre ha estado profundamente vinculada al desarrollo de la actividad turística, especialmente en lo que se refiere al traslado de bienes y personas (ALBALATE; BEL, 2010) así como a la existencia de infraestructura adecuada (por ejemplo, red carretera, aeropuertos, puertos marítimos, terminales de autobuses, etc.) que se entiende como una condición indispensable para el desenvolvimiento del turismo en cualquier territorio. La existencia de estas condiciones (flujo de personas e infraestructura) determinan la probabilidad de que más visitantes interactúen en diferentes destinos, expandiendo de esta forma los beneficios económicos y sociales derivados. A pesar de la gran relevancia de estos factores para el turismo, su estudio ha recibido una atención limitada, restringiendo así su comprensión (SHAW; WILLIAMS, 2002).

En lo que se refiere a la competitividad, la movilidad de turistas se ha convertido en un factor clave para el desarrollo y crecimiento tanto de destinos como regiones. En los últimos años se ha visto el nacimiento acelerado de una oferta renovada de destinos como resultado de la aplicación de nuevas estrategias de movilidad, promoción y comercialización. De esta forma, los turistas han tenido la posibilidad de conocer espacios y territorios alternativos a los destinos tradicionales. Lo anterior no solo ha generado curiosidad sino también interés por parte de distintas comunidades que desean participar en el mercado turístico, poniendo a disposición sus recursos naturales y culturales a potenciales visitantes. Estas comunidades identifican como indispensable desarrollar modelos y herramientas para el monitoreo y rastreo tanto de la movilidad como de las actividades de los visitantes de sus destinos. El objetivo principal es entender mejor los patrones de comportamiento espacial de los visitantes con el objeto de maximizar las oportunidades de las actividades turísticas y sus beneficios en las diferentes comunidades, construyendo así una ventaja competitiva y una diferenciación en el mercado.

La movilidad turística se promueve con el fin de diversificar experiencias turísticas, aumentar el número de visitantes, extender estancias, incrementar ingresos y gastos y al mismo tiempo involucrar una amplia variedad de destinos y proveedores turísticos en los servicios ofrecidos. El turismo representa un sector integrador de distintos servicios por lo cual su efecto socioeconómico es importante porque genera ingresos y empleos que se reparten entre establecimientos de distinto tamaño. En términos de su impacto geográfico, el turismo tiene la capacidad de encadenar ofertas novedosas en destinos nuevos y también consolidar a segmentos establecidos en zonas tradicionalmente turísticas. En un mundo globalizado con elecciones prácticamente ilimitadas, la competitividad de los destinos se vuelve imprescindible y siendo el turismo un servicio altamente fragmentado, requiere de la coordinación y colaboración de agrupaciones de actores involucrados en clústeres turísticos locales y regionales que conjunten varios destinos geográficamente cercanos, con el fin de consolidar imágenes, experiencias y marcas (LEMMETYINEN; GO, 2008). 
Un mejor entendimiento de la movilidad del turista es crucial para diseñar políticas públicas adecuadas de desarrollo turístico y mejorar la competitividad. No obstante su importancia, la literatura académica que estudia la emergencia de destinos alternativos como es el caso de los Pueblos Mágicos y en lo particular la movilidad de los turistas hacia estos territorios, es escasa y se concentra en los impactos sociales locales del programa (ver e.g., AARHUS-MÉNDEZ, RODRÍGUEZ, 2013; BALSLEV-CLAUSEN; GYIM-OTHY, 2015; GARCÍA-VEGA; GUERRERO-GARCÍA, 2014; MARTÍNEZ-RASCÓN, 2013; VELÁZQUEZ-GARCÍA, 2013). Con el objetivo de disminuir esta brecha de conocimiento, el presente trabajo pretende contribuir a comprender mejor la movilidad de los turistas en el territorio específico del Estado de Guanajuato, generando información relevante que, como se mencionó, es fundamental para la competitividad turística. En este trabajo, se identifican factores explicativos y predictivos acerca de la movilidad a través de la identificación de patrones. En contraste con trabajos anteriores, el análisis empleado en esta oportunidad es de carácter primordialmente cuantitativo a través del desarrollo de un modelo econométrico logístico.

\section{Problemas y objetivos de investigación}

Los enfoques tradicionales de investigación social no han logrado identificar plenamente los comportamientos referentes a la movilidad turística. Existen diversas limitaciones asociadas al diseño y aplicación de cuestionarios para indagar sobre el comportamiento de turistas en la planeación y ejecución de su movilidad, tomando en cuenta las opciones de transporte y la oferta de destinos turísticos en materia de atractivos, infraestructura y productos turísticos. Esta investigación pretende contribuir a comprender la movilidad turística entre destinos consolidados y emergentes en el contexto del Estado de Guanajuato, México. El programa de los Pueblos Mágicos obedece al objetivo de diversificar y ampliar la actividad turística, integrando nuevos destinos, segmentos y proveedores turísticos, aprovechando el potencial del turismo cultural a lo largo y al interior de este país (SECTUR, 2013). Evidentemente existe un dilema entre la generación de ingresos turísticos, su repartición entre actores locales y no locales y la sustentabilidad del desarrollo de localidades alejadas de los urbes principales y vulnerables (CLAUSEN, 2013). Contrario al modelo de turismo de sol y playa que lleva las actividades y servicios turísticos a sus clientes en el hotel o hasta la ribera donde se hospedan, en el segmento cultural es el cliente quien se mueve entre destino y destino, construyendo su experiencia turística con base en el consumo de lugares. Al promover el concepto de clústers, rutas y recorridos turísticos, mientras más favorablemente se alinean los factores geográficos de accesibilidad y distancia, con las ventajas iniciales de patrimonio y atractivos turísticos, y con la infraestructura y los productos turísticos diseñados, más factible resulta cualquier iniciativa para potencializar el turismo cultural. Los criterios de selección de nuevos destinos y el diseño de intervenciones es importante, porque el éxito de un programa multifacético de carácter y disperso geográficamente de entrada no está garantizado. En materia de movilidad, el artículo revela la experiencia local del programa, como referente para otros países latinoamericanos que pretenden adaptar un modelo similar e impulsar en sus propios contextos geográficos un turismo cultural como es el caso de Argentina, Chile, Ecuador, Guatemala, Perú entre otros. 


\section{El Programa de Pueblos Mágicos y el Estado de Guanajuato}

El Programa de Pueblos Mágicos fue creado de manera formal en el año 2001 y de acuerdo al criterio de la Secretaria de Turismo Federal (SECTUR) contribuye a revalorar a un conjunto de poblaciones del país que siempre han estado en el imaginario colectivo de la nación en su conjunto y que tienen el potencial de representar alternativas diferentes tanto para los visitantes nacionales como extranjeros. Actualmente son 111 los destinos que se encuentran inscritos dentro el Programa, de los cuales 37 fueron nombrados por el gobierno federal en el 2012 y los últimos 28 en 2015 (SECTUR, 2013, 2016).

Un Pueblo Mágico, de acuerdo a la SECTUR, se define como:

una localidad con una población base de 20,000 habitantes, con atributos simbólicos, leyendas, historia, hechos trascendentes, y cotidianidad. La localidad debe ubicarse en una distancia no mayor a los 200 kilómetros, o el equivalente a dos horas de distancia vía terrestre de un destino turístico consolidado o bien de una población considerada como mercado emisor (SECTUR, 2013).

De manera resumida, los objetivos del Programa son: 1) estructurar una oferta turística complementaria y diversificada hacía el interior del país, basada fundamentalmente en los atributos histórico - culturales de localidades singulares; 2) aprovechar la singularidad de las localidades para la generación de productos turísticos basados en las diferentes expresiones de la cultura local; 3) poner en valor, consolidar y/o reforzar los atractivos de las localidades con potencial turístico, fomentando así flujos turísticos que generen un mayor gasto en beneficio de la comunidad receptora (artesanías, gastronomía, amenidades y el comercio en general), así como la creación y/o modernización de los negocios turísticos locales; 4) que el turismo local se constituya como una herramienta del desarrollo sustentable de las localidades incorporadas al programa, así como en un programa de apoyo a la gestión municipal, y; 5) que las comunidades receptoras de las localidades participantes aprovechen y se beneficie del turismo como actividad redituable como opción de negocio, de trabajo y de forma de vida (SECTUR, 2013).

Es evidente que la mayoría de los objetivos del programa de Pueblos Mágicos apelan a un desarrollo turístico basado en la formación de redes y alianzas de cooperación turística.

Es importante mencionar que cinco de los 111 Pueblos Mágicos se encuentran en el Estado de Guanajuato (ver Mapa 1). En orden cronológico estos son: Dolores Hidalgo, nombrado en 2002, y Jalpa de Cánovas, Mineral de Pozos, Salvatierra y Yuriria, nombrados en 2012. De estos destinos se puede mencionar que solo Dolores Hidalgo es una comunidad con antecedentes turísticos, siendo "Cuna de la Independencia Nacional", un productor reconocido de artesanías de cerámica y el lugar natalicio de uno de los cantantes más emblemáticos del género de música "ranchera", además de ubicarse sobre la "Ruta de la Independencia" que pasa por la Sierra de Guanajuato y conecta a Guanajuato Capital con San Miguel de Allende, uno de los destinos emblemáticos del Estado. Por otro lado, Mineral de Pozos, un pueblo minero abandonado y recientemente repoblado, cuenta con cierto encanto y representa un destino turístico en formación, mientras que el resto de los destinos están en proceso de construir su oferta turística. Los casos de Jalpa de Cánovas, Salvatierra y Yuriria merecen un análisis aparte pues estas comunidades recién comienzan a experimentar el desarrollo turístico y hay pocos antecedentes locales sobre la dinámica de esta actividad. 
No hay duda que los cinco pueblos cuentan con un patrimonio cultural y natural aprovechable para fines turísticos, sin embargo, también es una realidad que todos requieren del diseño e implementación de programas adecuados de desarrollo que contemplen inversiones en atractivos e infraestructura complementaria ${ }^{1}$.

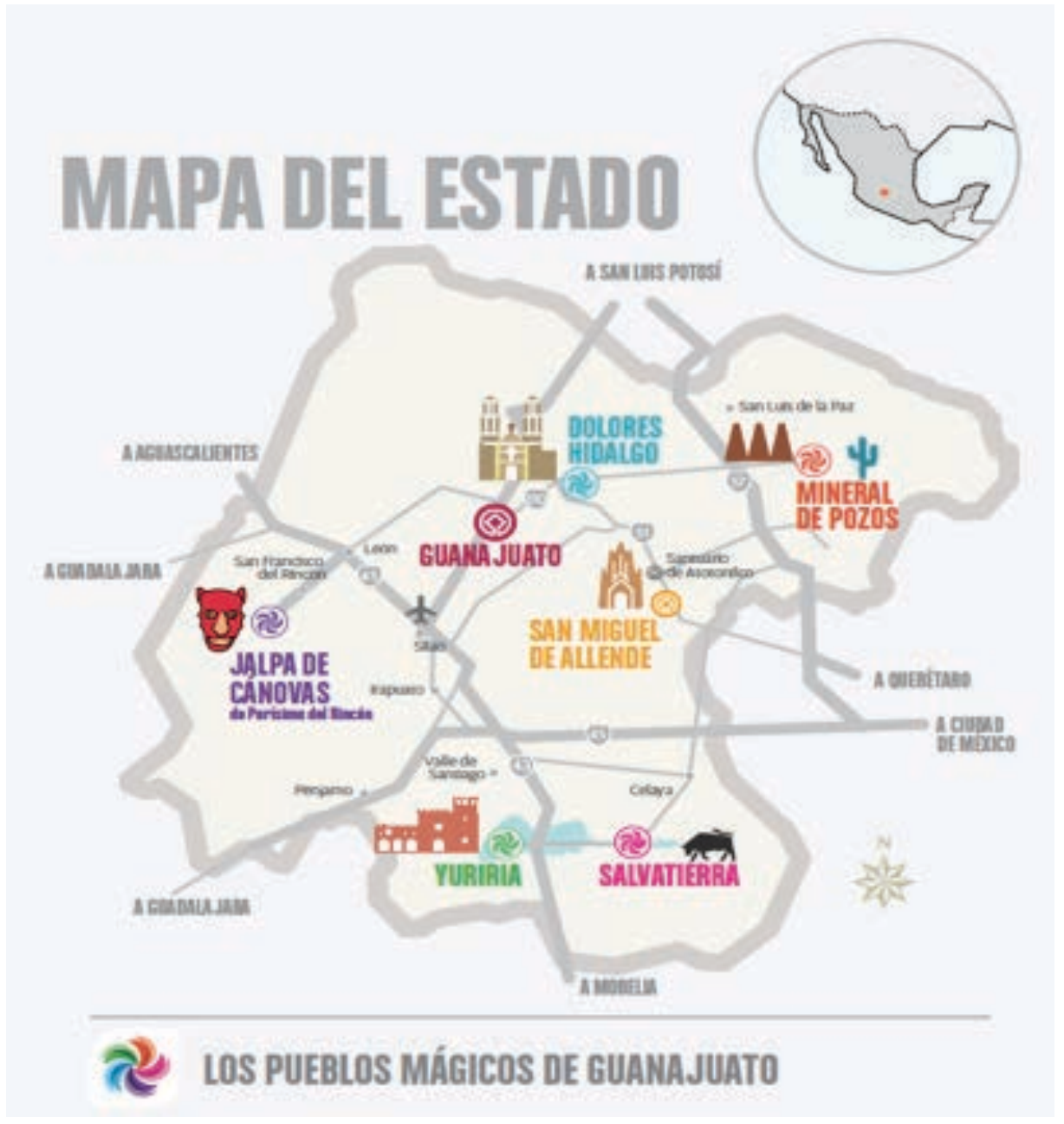

\section{Mapa 1 - Ubicación geográfica de los Pueblos Mágicos del Estado de Guanajuato.}

Fuente: Secretaría de Turismo del Estado de Guanajuato. http://www.gtoexperience.mx/pueblosmagicos/pueblos_guanajuato.pdf

\section{La competitividad turística}

La esencia del concepto de "competitividad" proviene de la utilización eficiente de los recursos para ofertar un producto o servicio, en este caso relacionado con el ámbito turístico, de mayor calidad en comparación con los demás ofertantes. De manera más concreta, la competitividad turística se puede definir como:

1 Conclusiones extraídas del estudio “Diagnóstico de Competitividad y Sustentabilidad de Pueblos Mágicos del Estado de Guanajuato, 2013” realizado por la Universidad de Guanajuato. 
La capacidad de los agentes que intervienen en la actividad turística de un país, de una región o de una zona para alcanzar sus objetivos por encima de la media del sector, de manera sostenible y sostenida; lo cual puede alcanzarse mediante la consecución de rentabilidades financieras por encima de la media en los ámbitos empresariales, y de rentabilidades sociales y ambientales como consecuencia de la actuación de organismos e instituciones públicas, así como consiguiendo la máxima satisfacción para los turistas, (SECALL, 2010, p. 7).

Como se puede apreciar, esta definición agrupa tres características principales: 1) el ámbito espacial; 2) el ámbito empresarial, y; 3 ) el ámbito social. El primer ámbito (espacial), se refiere a la necesidad de un territorio para desarrollar los destinos y las actividades turísticas que puedan generar beneficios sociales y económicos para las comunidades receptoras. Es en este espacio donde se generan una serie de productos y servicios dirigidos tanto a los visitantes como a los residentes. La segunda característica (empresarial), se trata de la organización de actores en la prestación de bienes y servicios para generar una ventaja comparativa respecto a otros territorios similares. El grado de compenetración entre estos actores sería hipotéticamente definitivo para determinar el nivel de competitividad de un destino. De este ámbito se desprenden los análisis de redes y clústeres que han tomado una fuerza importante en los últimos años (para una discusión más detallada ver SCOTT et al., 2008). Finalmente, la tercera característica (social) se refiere tanto al incremento de beneficios (económicos y/o sociales) derivados de un escenario más competitivo, así como la satisfacción de los visitantes en los destinos turísticos (SECALL, 2010).

\section{La dinámica turística del Estado de Guanajuato}

En la actualidad, los principales destinos turísticos con que cuenta el Estado de Guanajuato son: 1) la ciudad de León, con una vocación comercial y de negocios; 2) las ciudades patrimonio de la humanidad, Guanajuato Capital y San Miguel de Allende-Atotonilco, con una vocación cultural y; 3) los cinco Pueblos Mágicos, Dolores Hidalgo, Jalpa de Cánovas, Mineral de Pozos, Salvatierra y Yuriria, con una oferta incipiente de menor escala (ver Mapa 2).

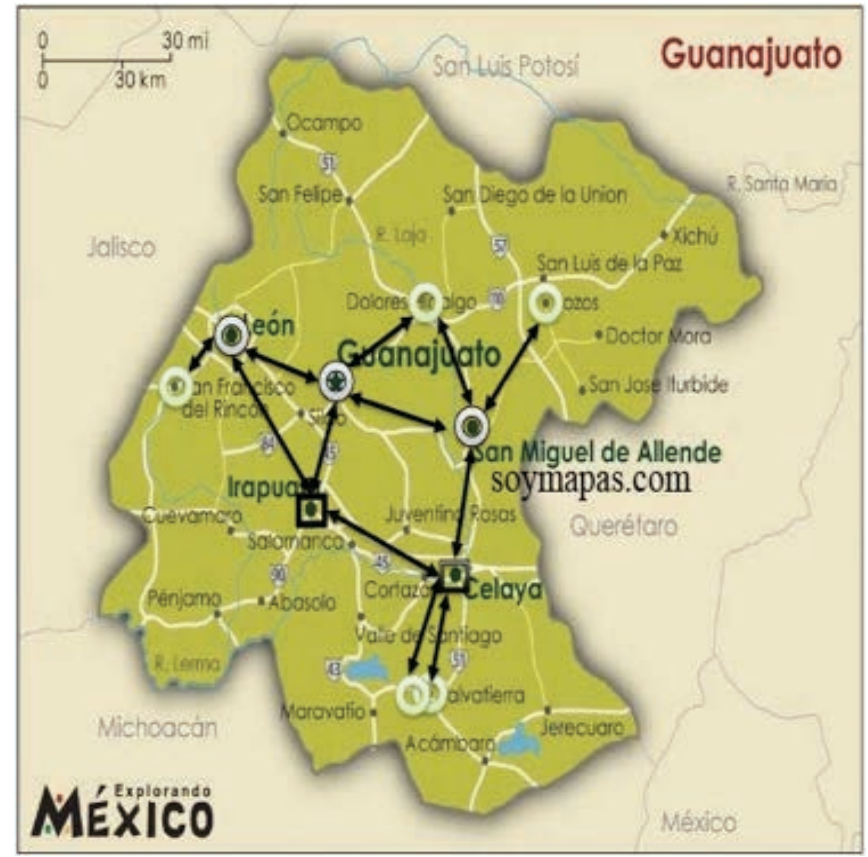

Mapa 2 - Dinámica turística del Estado de Guanajuato.

Fuente: Adaptado del sitio "Soy Mapas”.

http://soymapas.com/wp-content/uploads/2012/03/mapa-guanajuato.jpg 
Como se puede apreciar en el Mapa 2, los destinos más importantes se encuentran en la parte central del Estado (León, Guanajuato Capital y San Miguel de Allende). Estos tres destinos, concentran la mayor parte de la oferta turística y el flujo de visitantes. Los círculos en color claro representan los cinco Pueblos Mágicos que están dispersos geográficamente en el territorio del Estado. Así se puede apreciar, por ejemplo, que Jalpa de Cánovas tiene una cercanía con la ciudad de León, Dolores Hidalgo y Mineral de Pozos con Guanajuato Capital y San Miguel de Allende, y finalmente, Yuriria y Salvatierra con la ciudad de Celaya. Las ciudades de Irapuato y Celaya no son consideradas como destinos turísticos de importancia dado el flujo moderado de visitantes a estas localidades. Sin embargo, su importancia radica en la ubicación preferencial como puntos de acceso a los principales destinos turísticos del Estado. Este mapa ayuda a entender como la proximidad geográfica condiciona los flujos turísticos y el desarrollo de la actividad turística. En ese sentido, no es sorprendente que los Pueblos Mágicos del sur (Yuriria y Salvatierra), no presenten un nivel de desarrollo turístico comparable con Dolores Hidalgo, a pesar de representar una oferta de destinos conjunta.

De acuerdo con información del Observatorio Turístico del Estado de Guanajuato, las cifras más relevantes con respecto a la actividad turística a nivel Estatal se presentan en la Tabla 1.

Tabla 1 - Dinámica turística en el Estado de Guanajuato.

\begin{tabular}{|c|c|}
\hline \multicolumn{2}{|c|}{ Cifras de la actividad turística en el Estado de Guanajuato. } \\
\hline Concepto & Cifra \\
\hline Derrama económica & 72.030 Millones \\
\hline Visitantes & 22.2 Millones \\
\hline Inversión privada en turismo & 3.180 Millones (2013-2014) \\
\hline Número de circuitos turísticos & 5 \\
\hline Visitantes internacionales & 1.118 millones de visitantes \\
\hline Ocupación hotelera & $44.5 \%$ (Mayo 2015) \\
\hline
\end{tabular}

Fuente: Observatorio Turístico del Estado de Guanajuato (2015). http://www.observatorioturistico.org/

\section{Materiales y métodos}

Una forma de conceptualizar la movilidad es el proceso de decisión que hacen los turistas en relación al hospedaje y recorrido a destinos turísticos del Estado en combinación con su visita a un destino complementario, en este caso un Pueblo Mágico. La importancia que tiene este concepto es que permite diagnosticar la situación actual del sector así como hacer recomendaciones en el diseño de nuevos productos (rutas) en beneficio del desarrollo turístico. Como ya se mencionó en la introducción, la movilidad se concibe como un factor clave para la construcción de redes y alianzas de cooperación turísticas. 


\section{La base de datos}

La metodología que se diseñó en este estudio tiene una orientación primordialmente cuantitativa. Se utilizó una base de datos que se generó a partir de un levantamiento de información realizado en el año 2013 por una agencia de investigación de mercados contratada por la Secretaría de Turismo del Estado de Guanajuato (SECTUR-GTO, 2013). Se aplicaron un total de 900 encuestas personales a través de la técnica cara a cara dando respuesta a un total de 40 reactivos. El criterio de selección de los informantes fue el siguiente: para que una persona fuera entrevistada debía ser mayor de edad, haber estado al menos ya tres horas de antelación en el lugar de la entrevista, no trabajar ni estudiar en el destino y no residir en la ciudad. El instrumento contemplaba los siguientes apartados: 1) Filtro: cuatro preguntas en las que se establece si la persona entrevistada cumple con los criterios de selección; 2) Planeación de viaje; 3) Hábitos de viaje; 4) Gasto durante el viaje; 5) Nivel de satisfacción; 6) Lealtad al destino; 7) Medios de promoción y comercialización, y 8) Perfil sociodemográfico. Los puntos de muestreo fueron lugares de afluencia turística de los Pueblos Mágicos del Estado de Guanajuato.

\section{Las variables.}

En la sección de planeación de viaje se les preguntaba el propósito de su visita. Las respuestas tuvieron la siguiente distribución que se muestra en la Tabla 2.

Tabla 2 - Detalle de motivación.

\begin{tabular}{|c|c|}
\hline \multicolumn{2}{|c|}{ Propósito de viaje } \\
\hline Concepto & Porcentaje \\
\hline Luna de miel / romance & $1.2 \%$ \\
\hline Descanso/Vacaciones & $57.6 \%$ \\
\hline Visita a familiares /amigos & $20.7 \%$ \\
\hline Religión / peregrinación & $4.4 \%$ \\
\hline Negocios/ trabajo & $3.3 \%$ \\
\hline Congreso/Exposición & $0.9 \%$ \\
\hline Eventos (Deportivo, cultural, social) & $9.0 \%$ \\
\hline Compras & $1.7 \%$ \\
\hline Tratamiento de salud & $0.6 \%$ \\
\hline Viaje de estudios/ prácticas profesionales & $0.7 \%$ \\
\hline
\end{tabular}

Fuente: Elaboración propia basado en base de datos SECTUR-GTO (2013).

Como se puede apreciar, los casos más frecuentemente mencionados son "Descanso o Vacaciones" (57.6\%), "Visita a familiares o amigos" (20.7\%) y "Asistencia a eventos" (9\%). 
Para la construcción del modelo que se discute en la sección de "Resultados", se utilizaron variables dicotómicas para identificar cada una de las categorías de la variable Propósito de viaje. Estas son: Romance, Vacaciones, Visita.Familiares, Religión, Negocios, Congresos, Eventos, Compras, Salud, Prácticas. Profesionales. Es importante señalar que solo un número reducido de estas variables resultaron significativas.

En la sección de hábitos de viaje se les preguntó el número de noches que pernoctaría en el destino. La variable Hasta7noches identifica si el entrevistado respondió que durante su visita al estado, pernoctará de cero a siete noches.

De igual forma en la sección de hábitos de viaje se preguntaba el medio de transporte con el que el visitante llegó al destino turístico estudiado. La distribución se puede consultar en la Tabla 3.

\begin{tabular}{|c|c|}
\hline \multicolumn{2}{|c|}{ Medio de Transporte } \\
\hline Concepto & Porcentaje \\
\hline Automóvil propio & $72.2 \%$ \\
\hline Automóvil rentado & $1.4 \%$ \\
\hline Autobús & $23.1 \%$ \\
\hline Avión & $1.4 \%$ \\
\hline Autobús rentado & $1.2 \%$ \\
\hline Otro & $0.6 \%$ \\
\hline
\end{tabular}

Fuente: Elaboración propia basado en base de datos SECTUR-GTO (2013).

La variable dicotómica Automóvil indica si se contestó Automóvil propio. En forma sorpresiva, esta variable no fue significativa en ninguno de los modelos que se estimaron.

En la sección de perfil sociodemográfico se preguntó la ciudad de origen. Para el modelo que se discute en la sección de "Resultados", se definieron variables dicotómicas identificando cinco regiones de ciudad de origen de la siguiente forma:

1. Región-Internacional: Argentina, Brasil, Colombia, Estados Unidos, Inglaterra, Japón.

2. Región-Centro: Aguascalientes, Colima, Distrito Federal, Estado de México, Hidalgo, Jalisco, Michoacán, Morelos, Nayarit, Puebla, Querétaro, San Luis Potosí, Tlaxcala, Zacatecas.

3. Estado-Guanajuato.

4. Región-Norte: Baja California Norte, Baja California Sur, Chihuahua, Coahuila, Durango, Nuevo León, Sinaloa, Sonora, Tamaulipas.

5. Región-Sur: Campeche, Chiapas, Guerrero, Oaxaca, Quintana Roo, Tabasco, Veracruz.

A todos los entrevistados se les preguntó si visitarían otros destinos además del Pueblo Mágico, lugar de la entrevista. La variable Movilidad identifica cuando se respondió afirmativamente a la pregunta de si se consideran otros destinos incluidos dentro del viaje o se respondió "En OTRA ciudad" a la pregunta de dónde pernoctaría. 
Es importante señalar que mediante la base citada, la SECTUR-GTO caracterizó el Perfil del visitante a Pueblos Mágicos del Estado de Guanajuato (OTEG, 2013), documento que se encuentra disponible en su sitio web. Entre otros análisis, el documento reporta una lista comprensiva de estadísticas descriptivas en relación al comportamiento del visitante.

Para el análisis de la información contenida en la base de datos, se aplicó un modelo de regresión logística binomial. El trabajo de Hosmer e Lemeshow (2013) expone sistemáticamente los alcances y aplicaciones del modelo. Para los propósitos de este documento, basta decir que el modelo de regresión logística se escogió por su capacidad para predecir la probabilidad de movilidad tomando en cuenta un conjunto de factores relacionados al perfil del visitante así como su comportamiento de viaje.

\section{Resultados}

El modelo logístico permite establecer probabilidades condicionales para caracterizar la movilidad del visitante en función de factores sociodemográficos y hábitos de viaje. La pregunta genérica que motivó la especificación del modelo fue:

Dado que el visitante es proveniente de una región dada del país, se encuentra en uno de los Pueblos Mágicos del Estado de Guanajuato y no pernocta ahí, ¿Cuál es la probabilidad de que visite otro destino turístico del Estado?

La especificación del modelo LOGIT-PM es la siguiente:

$$
\text { Logit }(\text { Movilidad }=1 \mid x)=
$$

Intercepto $+d \_1$ Eventos $+d \_2$ Vacaciones $+d \_3$ PrácticasProfesionales +

d_4 RegiónCentro+d_5 EstadoGuanajuato+d_6 Hasta7noches.

Es decir, la probabilidad de que un visitante a alguno de los Pueblos Mágicos del estado haya respondido afirmativamente a la pregunta de si tiene planeado visitar otros destinos es explicada por factores sociodemográficos así como por factores de hábitos de viaje.

En la Tabla 4 se puede apreciar la estimación de los coeficientes y sus niveles de significancia en el modelo LOGIT-PM. Es importante señalar que en esta regresión otros propósitos de viaje resultaron ser no significativos y se eliminaron del modelo.

Tabla 4 - Estimación de coeficientes.

\begin{tabular}{|c|c|c|}
\hline \multicolumn{3}{|c|}{ Medio de Transporte } \\
\hline Variable & Coeficiente & z-Wald \\
\hline Intercepto & 1.36 & 0 \\
\hline Eventos & -2.05 & 0 \\
\hline Vacaciones & 0.82 & 0 \\
\hline Prácticas-Profesionales & 2.15 & 0.02 \\
\hline Región-Centro & -0.88 & 0 \\
\hline Estado-Guanajuato & -2.54 & 0 \\
\hline Hasta 7 Noches & -0.78 & 0.09 \\
\hline
\end{tabular}

Fuente: Elaboración propia basado en base de datos SECTUR-GTO (2013). 
La interpretación de los signos de los coeficientes es la siguiente: si un visitante viaja a un Pueblo Mágico por motivo de visitar un evento entonces habrá una menor probabilidad de que viaje a otros destinos en comparación con un visitante cuyo propósito sea tomar vacaciones o realizar prácticas profesionales. En relación al estado de origen, los visitantes locales del estado de Guanajuato tienen una menor probabilidad de movilidad en comparación de visitantes que vienen de la región centro del país. Otro factor que reduce la probabilidad de movilidad en los Pueblos Mágicos es la decisión de no pernoctar o quedar pocos días en el destino. Es posible formular pronósticos con el modelo. Por ejemplo, en la clase de entrevistas que contestaron que pernoctaban de 0 a 7 noches, la Tabla 5 presenta las probabilidades de movilidad.

Tabla 5 - Probabilidades de movilidad.

\begin{tabular}{|c|c|c|c|}
\hline \multicolumn{3}{|c|}{ Probabilidades de movilidad en función del propósito de viaje. } \\
\hline Variable & Estado-Guanajuato & Región-Centro & Otras-regiones \\
\hline Eventos & 0.02 & 0.09 & 0.19 \\
\hline Vacaciones & 0.24 & 0.63 & 0.8 \\
\hline Prácticas-Profesionales & 0.55 & 0.86 & 0.94 \\
\hline Complemento & 0.12 & 0.43 & 0.64 \\
\hline
\end{tabular}

Fuente: Elaboración propia basado en base de datos SECTUR-GTO (2013).

A partir de los datos de la Tabla 5, se puede pronosticar que si el origen del visitante es el Estado de Guanajuato y el propósito del viaje es "prácticas profesionales", la movilidad tiene una probabilidad de 0.55 . Quien visita Guanajuato con motivo de "vacaciones" o de "prácticas profesionales" y proviene de la región centro del país, entonces tiene una alta probabilidad ( 0.63 y 0.86 , respectivamente) de visitar más de un destino en su viaje. Si la región de origen es fuera del Estado de Guanajuato o del centro del país, entonces se pronostica un alto nivel de movilidad, con excepción única de que el propósito del viaje sea por "eventos" pues en este caso la probabilidad de movilidad es de 0.19 .

Existe una clara relación monótona de la movilidad con respecto a la distancia de la región de origen y el estado de Guanajuato. Lo anterior quiere decir que entre mayor es la distancia, más probable es la movilidad. En relación al propósito del viaje, se observa que visitar el Pueblo Mágico debido a la presencia de un evento reduce a casi cero la probabilidad de movilidad. Sin embargo, sugiere investigar la causalidad o correlación entre la celebración de eventos especiales y una mayor afluencia de turistas con una demanda creciente de hospedaje.

\section{Discusión}

En la sección de introducción se explicó la importancia de la movilidad en la competitividad de los destinos turísticos. De ahí la relevancia de la problemática planteada al inicio del trabajo de establecer sus determinantes. La competitividad en la industria de turismo es un elemento distinguido incorporado en la política pública. En efecto, el Plan Nacional de Desarrollo (PND) 2013 - 2018 describe en su eje "México Próspero" las acciones a seguir en el ramo turístico. De manera particular, se señala que es necesario "aprovechar el potencial turístico de México para generar una mayor derrama económica en 
el país", y plantea el interés por "Impulsar la innovación de la oferta y elevar la competitividad del sector turístico". México dispone de recursos excepcionales que consolidan una oferta incluyendo tanto a destinos tradicionales de sol y playa como a ciudades con diversos atractivos culturales y hasta comerciales. En el caso especial del Estado de Guanajuato, que es la entidad federativa analizada en el presente trabajo, se cuenta con repertorio diversificado en destinos con actividades culturales, comerciales y de contacto con la naturaleza, argumentos que indican la importancia de los determinantes de la movilidad. El impacto positivo de la movilidad se manifiesta también al estudiar el gasto realizado por el visitante, como indicador de la demanda turística. En efecto, los turistas nacionales que provienen de estados fuera de la región colindante con Guanajuato, gastan más (TREVIÑO et al., 2015) y según los resultados presentados en dicho estudio, poseen mayor movilidad visitando un mayor número de destinos durante su visita. En este sentido, el presente trabajo ha determinado una relación positiva entre distancia y movilidad. Es decir, entre más distante sea el lugar de origen del visitante y el Estado de Guanajuato, mayor será la probabilidad de que el turista realice un viaje multi-destino dentro del Estado pasando por un destino principal y una o más ciudades complementarias.

La formación de bloques dentro de una zona geográfica es una estrategia que permite a empresas chicas, medianas y grandes la oportunidad de competir a nivel global gracias a la información compartida, como es el acceso a investigación en marketing, innovaciones, etc. Alianzas de este tipo permitirían, teóricamente, alcanzar un nivel competitivo mayor que aquellos destinos o territorios donde la cooperación es baja o nula (MORIC, 2013). De tal suerte que en el sector turístico, las ventajas del patrimonio paisajístico, histórico y cultural representa una ventaja comparativa que se debe convertir en una ventaja competitiva a través de un círculo virtuoso de innovación e integración estratégica (BARROSO; FLORES, 2006). Lo anterior implica: 1) diseñar y conjuntar la infraestructura urbana y turística en armonía con el concepto turístico elegido (FORONDA; GARCÍA, 2009); 2) capacitar el capital humano responsable para el funcionamiento óptimo de tecnologías y calidad en el servicio (LILLO et al., 2007); 3) provocar una interdependencia entre el patrimonio, la identidad social y el desarrollo empresarial, involucrando a la ciudadanía en el proceso (SECALL, 2010); 4) aplicar macro-políticas para desarrollar eventos con inversiones significativas en productos innovadores y/o hasta icónicos (ALBERTI; GIUSTI, 2012), y; 5) crear una imagen del destino, alimentando creencias y conocimientos previos del individuo sobre el destino, con los sentimientos avivados a partir de una colección de experiencias fragmentadas respaldadas por múltiples actores locales durante su estancia (SAN MARTÍN et al., 2006).

Con respecto al tema de infraestructura, el Estado de Guanajuato cuenta con accesos terrestres importantes así como el Aeropuerto internacional del Bajío, el cual se encuentra situado en el municipio de Silao y representa un punto de entrada de visitantes importante. Sin embargo, en términos de facilidades, sería necesaria una revisión en diferentes rubros, por ejemplo, la cobertura de rutas aéreas. Aquí se observa un número reducido de conexiones directas, siendo comunes las escalas en la ciudad de México, que se mantiene como el principal centro receptor y distribuidor del país. También sería interesante revisar la oferta de productos estructurados en los que se pudieran ofrecer precios competitivos en vuelos y hospedaje que provoque una mayor afluencia de visitantes. Conjuntamente, se requiere complementar el esfuerzo estudiando la factibilidad de alternativas de transportación directa a ciudades principales.

Se puede visualizar que los destinos emergentes con mayor potencial de desarrollo son aquellos que se integran en ejes turísticos existentes por razones de cercanía geográfica, su afinidad temática o su naturaleza complementaria (ver Mapa 2). Cuatro de los cinco Pueblos Mágicos Guanajuatenses se encuentran en las orillas del Estado y de cara al desarrollo turístico se presentan nuevas oportunidades 
para la creación de infraestructura siendo el gran reto tomar decisiones adecuadas que produzcan mayores beneficios económicos y sociales.

Hay que mencionar, sin embargo, que el simple hecho de pertenecer al programa de Pueblos Mágicos no representa en ningún sentido una panacea de desarrollo o crecimiento económico. Un ejemplo de lo anterior es el caso de Dolores Hidalgo, que a pesar de su ubicación y relevancia histórica, aún no ha logrado consolidarse como destino turístico importante independientemente de los 14 años de su inclusión en el programa. En este sentido, el desarrollo turístico en los Pueblos Mágicos fuera del eje geográfico principal implica un esfuerzo de coordinación regional y el diseño de un plan conjunto que les permita tener un sentido de dirección. Lo que se puede observar actualmente en comunidades que presentan una nueva dinámica turística es que el impulso empresarial a nivel local es muy limitado y el involucramiento de la sociedad civil es incipiente. Por estas razones la política turística enfrenta el reto complejo de crear estrategias que impulsen la movilidad simultáneamente con el desarrollo y consolidación de productos turísticos de interés para la demanda real y potencial.

El modelo de movilidad desarrollado en esta investigación muestra que los turistas que provienen de destinos emisores lejanos o del extranjero tienen mayor propensión a la movilidad. Con respecto a los Pueblos Mágicos, estos patrones de movilidad se presentan mayormente entre Dolores Hidalgo, por sus atractivos y ubicación geográfica, y las ciudades de Guanajuato capital, León y San Miguel de Allende y en menor medida, entre Mineral de Pozos y San Miguel de Allende. Los demás Pueblos Mágicos están desarrollándose de manera limitada como destinos turísticos por lo cual la movilidad entre ellos es mínima. El reto de los destinos es lograr que los turistas que pernocten con familiares o amigos visiten por lo menos un destino complementario. Sin duda, la movilidad de los turistas depende también del motivo del viaje. Los visitantes que lleguen por motivos de trabajo (prácticas profesionales) tienen mayor propensión a la movilidad en tanto que los que vienen para participar en eventos no se desvían de su propósito principal. Existe el reto de convencer a los participantes de eventos que hagan una visita a un destino adicional, por ejemplo, al finalizar el evento.

En materia de estrategia, en primer lugar, se requiere fortalecer la alianza de Guanajuato capital, León y San Miguel de Allende, integrando al Pueblo Mágico de Dolores Hidalgo más plenamente como miembro de la misma, aprovechando su patrimonio cultural y cercanía geográfica. En segundo lugar, se deben fortalecer o desarrollar nuevas rutas y recorridos inspirados en las temáticas de la independencia, la plata, los monasterios, las ex-haciendas, las artesanías, los vinos etc., que conecten el clúster principal de Guanajuato capital y San Miguel de Allende con los Pueblos Mágicos con ofertas incipientes de Mineral de Pozos, Salvatierra, Yuriria y Jalpa de Cánovas. Se requiere crear conceptos innovadores para no reproducir solamente las temáticas tradicionales y fortalecer la marca de todos los destinos, incluyendo la marca Guanajuato Estado. Finalmente se requiere tomar en cuenta que mientras menos favorecido sea un pueblo en términos de su ubicación geográfica en relación a otros destinos consolidados, menos valioso su patrimonio y menos desarrollados sus atractivos y producto turístico, mayor será la intensidad de las iniciativas e inversiones requeridas para potencializar la "magia" del Pueblo Mágico para poder insertarlo dentro un clúster turístico. 


\section{Conclusión}

Recordando la observación realizada en la introducción acerca del número reducido de trabajos académicos dedicados al estudio de la movilidad hacia destinos alternativos como es el caso de los Pueblos Mágicos en México, el presente trabajo representa una contribución para reducir esta brecha de conocimiento. Alrededor de esta literatura, prácticamente no existen referencias en las que se utilice un enfoque cuantitativo, siendo mayor el énfasis en aspectos de tipo cualitativo. En este sentido, el presente trabajo innova a través de la aplicación de un modelo logístico para caracterizar la movilidad turística y entender cómo se visitan los Pueblos Mágicos del Estado de Guanajuato. Se ha demostrado la pertinencia del modelo al determinar los factores de mayor impacto para la movilidad, cuestión que puede ayudar a la toma de decisiones que abonen a fortalecer la competitividad turística del Estado. Este modelo también presenta aprendizajes que pueden ser valorados tanto en el programa Pueblos Mágicos en otras entidades de México así como para una posible aplicación a nivel internacional. Es una realidad que varios países de Centro y Sudamérica han considerado la posible aplicación del programa de Pueblos Mágicos, incluyendo Chile, Ecuador, Perú, Paraguay y Uruguay (EL ECONOMISTA, 2012; SECTUR, 2015). Con objetivos ostensiblemente parecidos, Buenas Aires arrancó el programa "Pueblos Turísticos" en 2008 con un enfoque de desarrollo de turismo comunitario (BUENOS AIRES TURISMO, sin fecha; CRUZ et al. 2014). El Salvador inició su programa de "Pueblos Vivos" en 2009 en la forma de un simple concurso, con planes de transformarse en un programa de desarrollo de turismo comunitario (CONTRAPUNTO, 2016; GÓMEZ, 2010; LA PRENSA GRÁFICA, 2015). Similar al programa de Pueblos Mágicos de México, desde 2010, Colombia cuenta con el programa "Nuestros Pueblos" constituidos dentro de la "Red de pueblos patrimonio de Colombia" e impulsados a través de promoción oficial, inversiones públicas en infraestructura y apoyos al emprendimiento (FONTUR, 2016; MCIT, 2016). Considerando el gran interés que ha causado la estrategia Mexicana de diversificación de la oferta turística a nivel internacional, los futuros estudios tienen el reto de descubrir más determinantes de la movilidad turística en el contexto de programas de turismo cultural como éste integrando cada vez a más comunidades a la dinámica turística. Finalmente, no se debe perder de vista que la implementación del Programa por si misma no garantiza el correcto desarrollo social o turístico de las comunidades receptoras. En todos los casos, existe la gran tarea de preparar adecuadamente a los destinos emergentes antes de exponerlos a la visita turística. Solo de esta forma se podrá garantizar que los beneficios del turismo en estos territorios sean mucho mayores a sus costos en los años por venir.

\section{Agradecimientos}

Se reconoce el apoyo de la SECTUR-GTO por permitir el uso de la información con la que se realizó el estudio empírico y por las diversas aportaciones de su personal que permitieron profundizar en el tema. De igual forma, se reconoce el apoyo de la Universidad de Guanajuato a través del proyecto 520/2015 "Análisis empírico del gasto, movilidad y competitividad en ciudades de Guanajuato." 


\section{Referencias}

ALBALATE, D.; BEL, G. Tourism and urban public transport: holding demand pressure under supply constraints. Tourism Management, v. 31, n. 3, p. 425-433, 2010.

ALBERTI, F. G.; GIUSTI, J. D. Cultural heritage, tourism and regional competitiveness. The Motor Valley cluster, City. Culture and Society, n. 3, p. 261-273, 2012.

AARHUS-MÉNDEZ, E.; RORÍGUEZ, S. Definiendo “lo mexicano". Una clave: persistencias del modelo urbano colonial en los “Pueblos Mágicos", Diálogos Latinoamericanos, n. 21, diciembre, 2013, p. 4667, 2013.

BALSLEV-CLAUSEN, H.; GYIM-OTHY, S. Seizing community participation in sustainable development: Pueblos Magicos of Mexico. Journal of Cleaner Production, in press, 2015.

BARROSO, M. O.; FLORES, D. La competitividad internacional de los destinos turísticos: del enfoque macroeconómico al enfoque estratégico. Cuadernos de Turismo, n. 17, p. 7-24, 2006.

BUENOS AIRES TURISMO. Pueblos Turísticos: programa de turismo comunitario, una apuesta al desarrollo turístico de las pequeñas localidades, sin fecha.

CLAUSEN H. B.; VELÁZQUEZ, M. A. El turismo cultural en Argentina, México y Brasil. Avances y desafíos. Diálogos Latinoamericanos 21, 2013.

CONTRAPUNTO. Diario Digital El Salvador, Mitur lanza Pueblos Vivos Multidestinos. Disponible en: 〈http://www.contrapunto.com.sv/archiv02016/economia/coyuntura/mitur-lanzo-pueblos-vivosmultidestinos>, 2015.

CRUZ, G. G. et al. Turismo y políticas públicas: análisis del programa Pueblos Turísticos y su implementación en Carlos Keen, Argentina. In: VI CONGRESO LATINOAMERICANO DE INVESTIGACIÓN TURÍSTICA, 25-27, 09-2014

EL ECONOMISTA. Replicarán programa de Pueblos Mágicos en el extranjero. Disponible en: 〈http:// eleconomista.com.mx/industrias/2012/09/16/replicaran-programa-pueblos-magicos-extranjero>, 2012.

FONTUR. Nuestros Pueblos. Disponible en: 〈http://www.pueblospatrimoniodecolombia.co/nuestrospueblos〉, 2016.

FORONDA, C.; GARCÍA, A. La apuesta por la calidad como elemento diferenciador en los destinos turísticos: planes renovados. Cuadernos de Turismo, n. 23, p. 89-110, 2009.

GARCíA-VEGA, D.; GUERRERO-GARCíA ROJAS, H. R. El programa Pueblos Mágicos: análisis de los resultados de una consulta local ciudadana. El caso de Cuitzeo, Michoacán, México. Economía y Sociedad, XVIII, n. 31, p. 71-94, 2014.

GÓMEZ I., ORTÍZ X. La apuesta por un turismo sostenible en El Salvador: propuestas para un cambio de políticas. Prisma, 2010.

HOSMER, D.; LEMESHOW, S. Applied logistic regression. 3rd edition. Wiley Series in Probability and Statistics, 2013.

LA PRENSA GRAFICA. Turismo premia a los ganadores del programa Pueblos Vivos. Disponible en: 〈http://www.laprensagrafica.com/2015/10/19/turismo-premia-a-los-ganadores-del-programapueblos-vivos〉, 2015. 
LEMMETYINEN, A.; GO, F. M. The key capabilities required for managing tourism business networks. Tourism Management, n. 30, p. 31-40, 2009.

LILLO, A.; RAMÓN, A.; SEVILLA, M. El capital humano como factor estratégico para la competitividad del sector turístico. Cuadernos de Turismo, n. 19, p. 47-69, 2007.

MARTínEZ-RASCÓN, C. Pueblos mágicos y nuevas ruralidades. El caso de Álamos, Sonora. Diálogos Latinoamericanos, n. 21, p. 31-45, 2013.

MINISTERIOS DE COMERCIO, INDUSTRIA Y TURISMO (MCIT). Pueblos Patrimonio Destinos de Turismo Cultural. Disponible en: 〈http://www.mincit.gov.co/publicaciones.php?id=2156〉. Acceso en: 23/02/2012.

MORIC, I. Clusters as a Factor of rural Tourism Competitiveness: Montenegro Experiences. Business Systems Research, n. 4, p. 94-107, 2013.

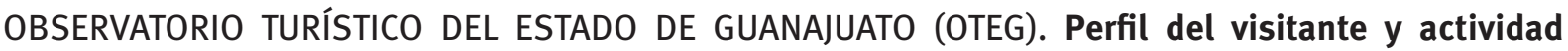
turística a Pueblos Mágicos del Estado de Guanajuato, 2013.

Tablero de control para medir la actividad turística del Estado de Guanajuato, 2015.

OLIVERI, A. M.; PARROCO, A. M.; VACCINA, F. Tourist Mobility and Destination Competitiveness. Rivista Italiana di Economia Demografia e Statistica, v. 66, n. 2, p. 213-234, 2012.

PLAN NACIONAL DE DESARROLLO (PND). Plan Nacional de Desarrollo 2013-2018, Gobierno de la República, México, 2013.

SAN MARTÍN, H.; RODRÍGUEZ, I.; VÁZQUEZ, R. Análisis de la imagen en turismo mediante técnicas estructuradas y no estructuradas: implicaciones competitivas para los destinos turísticos. Revista Asturiana de Economía, n. 35, p. 69-91, 2006.

SCOTT, N.; BAGGIO, R.; COOPER, C. Network analysis and tourism. From theory to practice, Clevedon, Nueva York y Ontario, Channel View Publications, 2008.

SECALL, R. E. La competitividad de las zonas turísticas. Málaga: Facultad de CC. EE. y EE. Universidad de Málaga, 2010.

SECTUR. Diagnóstico de Competitividad y Sustentabilidad de Pueblos Mágicos del Estado de Guanajuato, 2013. Universidad de Guanajuato, 2013.

. Organiza SECTUR seminario sobre Pueblos Mágicos a delegaciones de Centro y Sudamérica. Boletín 143, 2015. Disponible en: 〈http://www.sectur.gob.mx/sala-de-prensa/2015/o8/17/boletin143-organiza-sectur-seminario-sobre-pueblos-magicos-a-delegaciones-de-centro-y-sudamerica/>, 2016.

Pueblos Mágicos: herencia que impulsan Turismo. Disponible en: 〈http://www.gob.mx/ sectur/articulos/pueblos-magicos-herencia-que-impulsan-turismo〉, 2016.

SECTUR-GTO. Estudio de Perfil de los Visitantes a los Pueblos Mágicos, 2013.

SHAW, G.; WILLIAMS, A. M. Critical Issues in Tourism: a geographical perspective. Wiley, 2002.

SHOVAL, N.; ISSACSON, M. Tourist mobility and advanced tracking technologies. Nueva York y Oxon, Routledge. Taylor and Francis, 2010. 
TREVIÑO-AGUILAR, E.; HEALD, J.; GUERRERO, R. Un modelo del gasto con factores sociodemográficos y de hábitos de viaje en Pueblos Mágicos del Estado de Guanajuato. Investigaciones Turísticas (en imprenta), 2015.

VELÁZQUEZ-GARCíA, M. A. La formulación de las políticas públicas de turismo en México. El caso del programa federal "Pueblos Mágicos" 2001-2012. Diálogos Latinoamericanos, n. 21, p. 89-110, 2013. 